\title{
Chromatographic Fingerprint Analysis and Bioactivity of Origanum Vulgare Extracts
}

\author{
Corina Bubueanu ${ }^{*}$, Ioana Nicu ${ }^{2}$, Lucia Pirvu ${ }^{3}$ \\ 1,3Extractive Biotechnologies, National Institute for Chemical Pharmaceutical Research and Development (ICCF Bucharest), Vitan Avenue- 112, \\ Sector 3, Bucharest, ROMANIA \\ ${ }^{2}$ Pharmacology Department, National Institute for Chemical Pharmaceutical Research and Development (ICCF Bucharest), Vitan Avenue- 112, \\ Sector 3, Bucharest, ROMANIA
}

*Correspondence (Email):

corina.bubueanu@yahoo.com

\begin{abstract}
A very important measure for any country, in terms of public health problems and commercial exploitation, must be the development of medicinal and aromatic plant resources. Long-term strategies for the conservation of medicinal and aromatic plants, personnel training, cultivation or passage in culture of valuable species, sustainable methods of harvesting and processing plants to promote scientific research and validation of traditional remedies are needed. In the present, the natural compounds research are mainly focused on their role in improving health. The extraction processes of the natural compound are significant both in scientific and economic terms. The goal is to obtain extracts with pharmacological activity with a minimum production cost.

In this paper are analyzed hot and cold ethanol $50 \%$ extracts of Origanum vulgare (Fam. Lamiaceae) species in terms of qualitative and quantitative chemical composition, and their antioxidant and antimicrobial potential.

The results obtained showed no significant differences in terms of chemical composition and bioactivity, between the extracts. Thus, in industry sector these results can be used for reducing cost production.
\end{abstract}

Key words: hot extract, cold extract, Origanum vulgare, industry

This article is is licensed under a Creative Commons Attribution-NonCommercial 4.0 International License.

Attribution-NonCommercial (CC BY-NC) license lets others remix, tweak, and build upon work non-commercially, and although the new works must also

acknowledge \& be non-commercial.

\section{INTRODUCTION}

Development of medicinal and aromatic plant resources for both commercial exploitation and to meet the needs of the population in terms of health problems must be a measure of utmost importance for any country.

Origanum vulgare (Lamiaceae family) is in an aromatic and medicinal species used both in phytopharmaceutical and food industry. In food preparations for his flavor. In the phytopharmaceutical industry for respiratory and stomach ailments and for antimicrobial and antioxidant properties, being present in many herbal products. O. vulgare is an important source of essential oil and phenolic metabolites. The essential oil are extracted from the aerial parts of Origanum vulgare, especially from the flowers. Obtained by steam distillation the essential oil contain (thymol, carvacrol, $\beta$-fenchyl alcohol and $\delta$-terpineol $\_$-cymene, $\gamma$-terpinene, caryophyllene, spathulenol, germacrene-D) (Teixeira, 2013; Radusiene, et al 2008; Parvu, 1997).

In water or alcohol extracts are presents phenolic compounds. Antioxidant activity is mainly related to these compounds (Cervato et. al. 2000; Bendini, et. al. 2002).

This paper shows obtaining methods for various extracts of Origanum vulgare species, analyzing the qualitative and quantitative chemical composition, and their antioxidant and antimicrobial potential. 


\section{Materials AND Methods}

Chemicals: Folin-Ciocalteu's Phenol reagent, 2.2-Di(4-tert-octyphenyl)-1-picrylhydrazyl (DPPH), caffeic acid, chlorogenic acid, rutin, were purchased from Sigma Chemical Co., St. Louis, USA, NP - natural product (Fluka), PEG polyethylene glycol (Fluka), methanol and ethanol HPLC grade, ethyl acetate, acetic acid, formic acid, sulfuric acid, sodium molybdate, ammonium phosphate., Staphylococcus aureus ATCC 6538, Candida albicans ATCC 10231, Escherichia coli ATCC 8739 test organisms were purchased Merck Romania S.R.L. Origanum vulgare L. dried vegetal material was purchased from SC Anima Plant SRL, Romania.

Preparation of extracts - The aerial parts of Origanum vulgare has been extracted with ethanol $50 \%$, vegetal material/ solvent rate $-1 / 20 \mathrm{~m} / \mathrm{v}$ at the boiling temperature of the solvent/at room temperature, for 2 hours under continuous stirring. After filtration, the solution was concentrated under reduced pressure $(72-74 \mathrm{~mm} \mathrm{Hg})$ and $50^{\circ} \mathrm{C}$ to a vegetal material/ethanolic extract rate $-1 / 5(\mathrm{~m} / \mathrm{v})$. The insoluble substances were removed by centrifugation at $3000 \mathrm{rpm}$ for 30 minutes. The resulted solution was spray-dried.

HPTLC Analysis for Polyphenols: The method have been performed according to TLC Atlas - Plant Drug Analyses (Wagner and Bladt, 1996). The characteristic fingerprint profile of the extracts was performed by a densitometric HPTLC analysis. 2-3 $\mu \mathrm{l}$ of the samples (extracts were dissolved with HPLC grade $50 \%$ (v/v) methanol to $1 \%$ (m/v) solution concentration) and $1-1 \mu \mathrm{l}$ of references substances (rutin, chlorogenic acid, caffeic acid, $10^{-3} \mathrm{M}$ - Sigma-Aldrich) were loaded as $10 \mathrm{~mm}$ band length in the 20x10 Silica gel $60 \mathrm{~F}_{254}$ TLC plate. The spotting was made using Hamilton- Bonaduz, Schweiz syringe and CAMAG LINOMAT 5 instrument. Ethyl acetate-acetic acid-formic acid-water 100:11:11:27 (v/v/v/v) was the mobile phase. After development, plates were dried and derivatised in NP-PEG reagent. The evaluation of the fingerprints was made at 366nm in fluorescence mode with a WinCats and VideoScan software.

Quantitative analytical determination- Total phenol content was determined with Folin - Ciocalteu method (European Pharmacopoeia 6,0). Briefly, $1 \mathrm{ml}$ of extract was transferred to a $25 \mathrm{ml}$ volumetric flask, $10 \mathrm{ml}$ of water and $1 \mathrm{ml}$ of Folin Ciocalteu reagent has been added. The volume has been made to $25 \mathrm{ml}$ with $5 \%$ sodium carbonate (w/v). The blend has been lefted at room temperature for 30 minutes. Then the absorbance of the samples was read at $760 \mathrm{~nm}$ with a UV/VIS spectrophotometer (Helios $\lambda$, Thermo Electron Corporation). Distilled water was used as a blank. Total phenol content was determined from the extrapolation of the calibration curve $(y=1,474 x-0,438, R 2=0.992)$, which was obtained for gallic acid. The results were expressed as milligrams of gallic acid equivalents (GAE) per 100 gram.

Free radical scavenging assay. $50 \mu \mathrm{l}$ aliquots of the extract (concentration were $1 \%, 0.1 \%, 0.01 \%$ and $0.001 \%$ in methanol) were mixed with $2950 \mu \mathrm{l}$ of the methanolic DPPH solution $(0.025 \mathrm{~g} / \mathrm{L})$. The radical scavenging activity of the extracts against 2,2-Diphenyl-1-picrylhydrazyl radical (Sigma-Aldrich) was determined by measuring UV absorbance at $517 \mathrm{~nm}$ (Sanchez-Moreno, et.al. 1998). A blank solution was prepared containing the same amount of methanol and DPPH and measured after standing at r.t. 30 minutes. Caffeic acid methanolic solution (1\%) was used as positive controls.

The radical scavenging activity was calculated using the following formula:

$\%$ inhibition $=\{(\mathrm{AB}-\mathrm{AA}) / \mathrm{AB}\} \times 100$.

Where $\mathrm{AB}$ is the absorption of blank sample and AA is the absorption of tested extract solution.

The total antioxidant capacity assay was evaluated by phosphomolybdenum method, according to Prieto et al. (Prieto, et. al. 1999). Concentration of the extracts tested were $0.1 \%, 0.01 \%$ and $0.001 \%$ in ethanol. To 0.3 ml ethanolic solution of the sample has been added $2.7 \mathrm{ml}$ of reagent solution $(0.6 \mathrm{M}$ sulfuric acid, $28 \mathrm{mM}$ sodium molybdate, and $4 \mathrm{mM}$ ammonium phosphate). The mixtures have been incubated at $95^{\circ} \mathrm{C}$ for 90 minutes. After cooling the samples to room temperature, their extinction was measured at $695 \mathrm{~nm}$ at UV-VIS spectrophotometer. Ethanol has been used as negative control. The antioxidant capacity has been expressed as ascorbic acid equivalent to $1 \mathrm{mg}$ of active substance. The calibration curve is linear for ascorbic acid in the range of 0.001 to $1 \mathrm{mg} / \mathrm{ml}, \mathrm{n}=6, \mathrm{r}^{2}=0.999$.

Antimicrobial assay. The tests were carried out using cylinder method in plates on three microorganisms: a Gramnegative bacterium (Escherichia coli ATCC 8739), a Gram-positive bacterium (Staphylococcus aureus ATCC 6538) and a fungus (Candida albicans ATCC 10231). Antimicrobial activity was calculated on basis of the diameter of the growth inhibition as follows: (<10 mm) - no antimicrobial activity; (10-15 mm) - weak antimicrobial activity; (16-20 mm) moderate antimicrobial activity; $(>20 \mathrm{~mm})$ - certain antimicrobial activity. Statistical Analysis. The experiments were repeated three times. The results have been expressed as means \pm S.D, and the difference was tested by Student's t-test.

\section{RESULTS}

Extractions were performed similarly, the only difference being the temperature of extraction. Thus, from the same amount of raw material, for the extraction performed at room temperature have been obtained 3.8g atomized extract (Origanum vulgare C - OVC), and for the extraction carried out at the reflux temperature of the solvent, have been obtained $4.3 \mathrm{~g}$ atomized extract (Origanum vulgare $\mathrm{H}-\mathrm{OVH}$ ). Both were yellow-brown, hygroscopic extracts, with characteristic taste and odor.

HPTLC profile of the extracts has been recorded in Figure 1. Blue and yellow fluorescent spots detected at 366nm after derivatization of the chromatogram confirms the presence of polyphenols. 
HPLTC profile of the two extracts is the same. There are a number of 5 principal spots revealed in the chromatogram for both extracts. Caffeic acid (Rf 0.96), is identified compared with reference substance. The P9-P5 spots are probably apigenin and luteolin derivates and P2 -P3 blue spots - are most likely izochlorogenic acids (Wagner and Bladt, 1996).

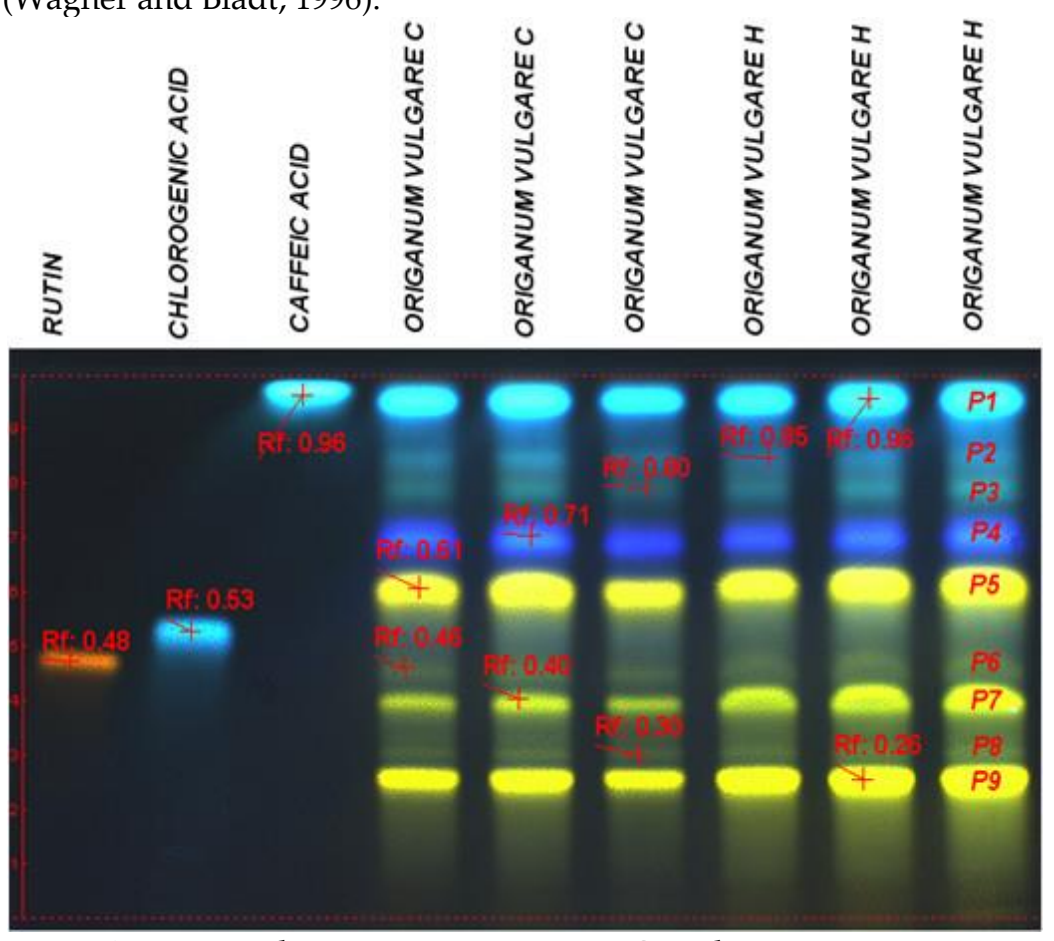

Figure 1: Extracts chromatogram in HPTLC analysis

The results of the Folin-Ciocalteu total phenols assay content are reported in Table 1.

Table 1: Quantitative composition of extracts

\begin{tabular}{|l|c|}
\hline Extracts & Total phenols content (expressed as gallic acid equivalents) (mg/100g) \\
\hline Origanum vulgare $C$ & 2,87 \\
\hline Origanum vulgare $H$ & 2,31 \\
\hline
\end{tabular}

\section{DPPH assay}

DPPH (2,2-diphenyl-1-picryl-hydrazyl-hydrate) (stable at room temperature) is an antioxidant assay based on electron-transfer that produces a violet solution in ethanol or methanol. DPPH is an easy and rapid method for the evaluation of antioxidants by spectrophotometry (Huang, et al. 2005).

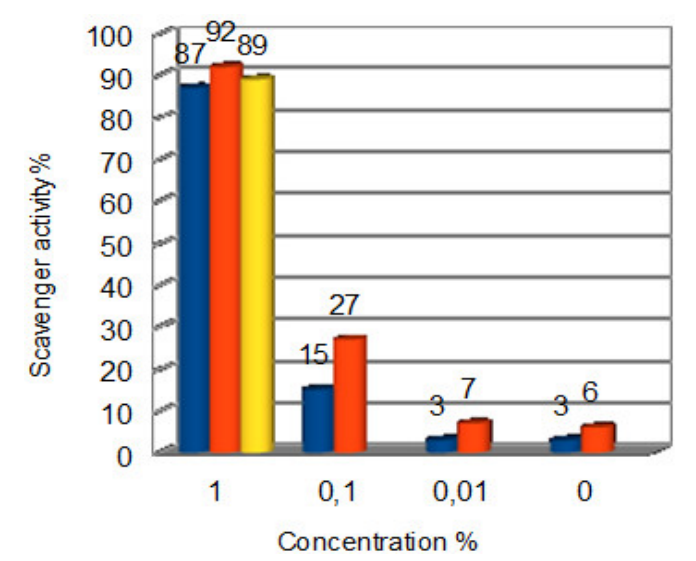

The potential of the free radical scavenger DPPH was evaluated for Origanum vulgare $C$ and Origanum vulgare $H$ extracts at concentrations $(1 \mathrm{mg}$ / $\mathrm{ml}, 0.1 \mathrm{mg} / \mathrm{ml}, 0.01 \mathrm{mg} / \mathrm{ml}$ and $0.001 \mathrm{mg} / \mathrm{ml}$ ) and also for reference substance caffeic acid $(1 \mathrm{mg} /$ $\mathrm{ml}$ ) (Figure 2). All evaluations were performed in triplicate, obtaining the average values of the inhibitory capacity for extracts of the stable radical with the nitrogen. The scavenger activities of the extracts (Origanum vulgare C -OVC $-87 \pm 0.10 \%$ and Origanum vulgare $\mathrm{H}-\mathrm{OVH}-92 \pm 0.11 \%$ ) are comparable with the one of the caffeic acid $(89 \pm 0.19 \%)$.

Figure 2: Scavenger activity of the extracts comparative with references substance 


\section{Total antioxidant capacity assay}

One of the methods that has been used for testing the capacity of the antioxidant to participate in the oxidationreduction reaction as a reducing is the total antioxidant capacity assay. This method is capable to determinate the

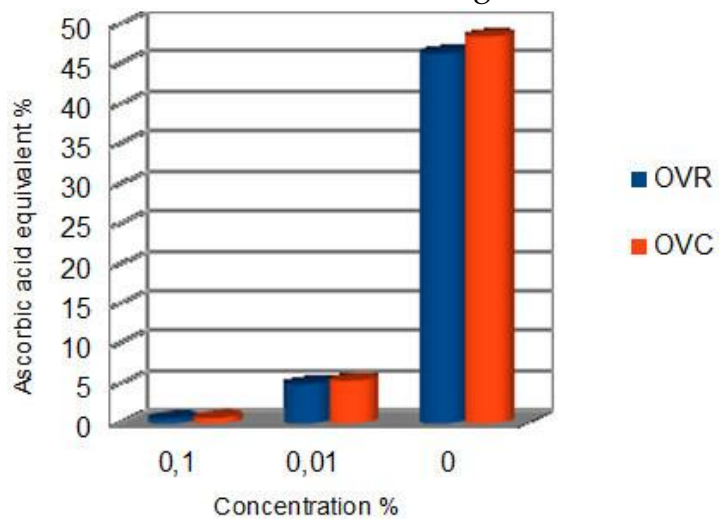
antioxidant activity by the reduction reaction of the reagent solution (0.6 M sulfuric acid, $28 \mathrm{mM}$ sodium molybdate, and 4 $\mathrm{mM}$ ammonium phosphate). The method shows, indirectly the ability to reduce the radicals of the antioxidant tested, by the mechanism of electron donor.

The results of the antioxidant capacity assay has been shown in Figure 3. Both extracts have strong antioxidant activities. The results obtained are similar. Origanum vulgare $\mathrm{H}$ extract $-\mathrm{OVH}$ at $0.1 \mathrm{mg} / \mathrm{ml}$ concentration have an antioxidant capacity equivalent to $0.65 \pm 0.06 \%$ ascorbic acid and Origanum vulgare $\mathrm{C}$-OVC at the same concentration - equivalent to $0.69 \pm 0.09 \%$ ascorbic acid.

Figure 3: Total antioxidant capacity of the extracts

Antimicrobial assay. The results obtained by the calculated diameter of the growth inhibition $(<10 \mathrm{~mm})-$ showing that the extracts have no antimicrobial activity.

\section{Discussions}

The results show that both qualitative and quantitative differences and antioxidant and antimicrobial activities regarding the two extracts are virtually nonexistent. Studies on species Origanum vulgare are heading mainly to the obtaining and characterization and pharmacological potential of the essential oil (Miller, et al. 2014; Mancini, et al. 2014). In terms of hydro-alcoholic extracts, studies are mainly about the quantity and quality content. Water extracts (obtained by maceration of dry plant material, or boiling water for $3 \mathrm{~h}$; or water for 3 days at room temperature) and ethanolic extract (obtained with ethanol extraction for 3 days at room temperature) have similar results only for the content in polyphenols for hot water extract (Teixeira,et al. 2013). The difference may be probable because of the extraction technique. In this experiment, having only one variable in the technological parameters, the differences are easier to be observed. Thus, this extracts being almost the same, in the terms of qualitative and quantitative composition, and in antioxidant and antimicrobial activities, is important to know that the resources used in the obtaining process of them, can be reduced.

\section{CONCLUSIONS}

Given the use of extracts obtained from Origanum vulgare species in many preparations with therapeutic value, the results presented in this paper, respectively the obtaining method, can be used at industrial level with significant reductions in production costs, while obtaining products with superior therapeutics value.

\section{REFERENCES}

Bendini A., Gallina T. Toschi and G. Lercker 2002. Antioxidant activity of oregano (Origanum vulgare L.) leaves, Ital. J. Food Sci. n. 1, vol. 14 Cervato Giovanna, Carabelli Marta, Gervasio Silvia, Cittera Andrea, Cazzola Roberta and Cestaro Benvenuto, 2000. Antioxidant properties of Oregano (Origanum vulgare) leaf extracts- Journal of Food Biochemistry 24, 453-465;

European Pharmacopoeia 6,0

Huang DJ, Ou BX, Prior RL. 2005 The chemistry behind antioxidant capacity assays. J Agric Food Chem;53:1841-1856

Mancini E, Camele I, Elshafie HS, De Martino L, Pellegrino C, Grulova D, DeFeo V, 2014. Chemical composition and biological activity of the essential oil of Origanum vulgare ssp. hirtum from different areas in the Southern Apennines (Italy).Chem Biodivers. Apr;11(4):639-51

Miller AB, Cates RG, Lawrence M, Soria JA, Espinoza LV, Martinez JV, Arbizú DA, 2014. The antibacterial and antifungal activity of essential oils extracted from Guatemalan medicinal plants.Pharm Biol. Oct 21:1-7;

Parvu Constantin 1997. Universul plantelor Mica enciclopedie - Ed. Enciclopedica , Bucuresti

Prieto P., Pineda M., Aquilar M. 1999. Spectrophotometric quantitation of antioxidant capacity through the formation of a phosphomolybdenum complex: specific application to the determination of vitamin E. / Analytical Biochemistry, p. 337-341

Radusiene Jolita, Ivanauskas Liudas, Janulis Valdimaras, Jakstas Valdas, 2008. Composition and variability of phenolic compounds in Origanum vulgare from Lithuania. Biologija. Vol. 54. Nr. 1. P. 45-49;

Sanchez-Moreno, C. Larrauri, J.A., Saura-Calixto, F., 1998. A procedure to measure the antiradical efficiency of polyphenols. Journal of Agricultural and Food Chemistry 76, 270-276

Teixeira Barbara, Antonio Marques, Cristina Ramos, Carmo Serrano, Olivia Matos, Nuno R Neng, Jose M. F. Nogueira, Jorge Alexandre Saraiva and Maria Leonor Nunes, 2013. Chemical composition and bioactivity of different oregano (Origanum vulgare) extracts and essential oil. J Sci Food Agric 93 (11): 2707-2714;

Wagner H., Bladt S., 1996. Plant Drug Analysis, Second Edition, Springer.

$$
\text { -- } 0 \text {-- }
$$

